\section{$\beta$-caténine et contrôle de la prolifération des cellules intestinales normales et cancéreuses}

Richard Hamelin, Alex Duval
Inserm U.434, CEPH,

27, rue Juliette-Dodu,

75010 Paris, France.

richard.hamelin@cephb.fr

alex.duval@cephb.fr
> La voie de signalisation wnt/wingless joue un rôle important au cours du développement embryonnaire en modulant l'expression de signaux intercellulaires qui contrôlent la croissance, la migration, le déterminisme et la polarisation cellulaires [1]. Elle a été particulièrement étudiée au cours du développement chez le nématode, la drosophile et les amphibiens, mais ses constituants sont très conservés chez les vertébrés. Dans les tissus adultes, elle participe au contrôle de la prolifération cellulaire. Une activation anormale de cette voie par mutation de l'un ou l'autre de ses composants joue également un rôle important dans un grand nombre de cancers humains [2]. Cette voie est alors plus couramment appelée voie APC (adenomatous polyposis coli)/ $\beta$-caténine/ TCF ( $T$-cell factor).

Que ce soit au cours du développement ou de la carcinogenèse, la clé de voûte de cette voie est la $\beta$-caténine: lorsque la voie est inactive, celle-ci est dégradée, lorsque la voie est active, elle est libérée dans le cytoplasme où elle exerce ses fonctions [2]. Dans la position «OFF», la $\beta$-caténine (ou Armadillo chez la drosophile) est partie intégrante d'un complexe multiprotéique composé de l'Axine (ou son homologue, la conductine), de la protéine phosphatase $P P 2 A$, de la glycogène synthase kinase $3 \beta$ (GSK3 $\beta$ ) et d'APC. Au sein de ce complexe, l'interaction entre l'Axine et la GSK3 $\beta$ semble favoriser la phosphorylation de la $\beta$-caténine, processus qui permettra l'ubiquitinylation de la $\beta$-caté- nine et sa dégradation ultérieure par la voie du protéasome. La position « $O N »$ est obtenue de manière différente selon qu'il s'agit d'un processus normal du développement ou d'un processus de carcinogenèse. Dans le premier cas, l'activation des récepteurs frizzled par la fixation de Wnt conduit à la phosphorylation de la protéine dishevelled qui, par son association à l'Axine, empêche la GSK3 $\beta$ de phosphoryler ses substrats parmi lesquels se trouve la $\beta$-caténine, rendant ainsi la liberté à cette dernière. Dans les cancers, indépendamment de toute activation du récepteur frizzled, c'est l'inactivation, ou l'activation, des gènes codant pour des protéines impliquées dans le complexe phosphorylant la $\beta$-caténine qui bloque la dégradation de la $\beta$-caténine. Le plus souvent, il s'agit de mutations de APC mais il peut aussi s'agir de mutations au niveau de séquences répétées codantes du gène codant pour l'Axine, ou celles, activatrices, dans l'exon 3 du gène codant pour la $\beta$-caténine au niveau des sites de phosphorylation.

Dans tous les cas, lorsqu'elle n'est pas dégradée, la $\beta$-caténine s'accumule dans le cytoplasme. Son rôle dans l'adhérence cellulaire par son association avec la cadhérine $\varepsilon$ et l'actine du cytosquelette pour former les jonctions d'adhérence (gap jonction) ne sera pas discuté ici. Une autre fraction de la $\beta$ caténine libre est véhiculée dans le noyau où elle s'associe aux facteurs de transcription TCF/LEF, modulant ainsi leur activité et activant (ou inactivant) l'expression d'un grand nombre de gènes cibles $[3,4]$. c-MYC est l'un d'entre eux, et le premier gène répondant à une telle régulation [5]. Une liste de gènes dont l'expression est contrôlée par le complexe $\beta$-caténine/TCF peut être consultée sur http://www.stanford.edu/ rnusse/wntwindow.html.

Plus récemment, $M$. van de Wetering et al. ont établi une nouvelle liste de gènes cibles de cette voie en réalisant une analyse différentielle d'expression par hybridation sur des puces à ADN, à partir de deux lignées de cancers colorectaux, LS174T et DLD-1 dans lesquelles une mutation activatrice de la $\beta$-caténine, ou une mutation inactivatrice d'APC, activent la voie $A P C / \beta$-caténine/TCF [6]. La position «OFF» de la voie APC/ $\beta$ caténine/TCF a été obtenue dans les deux cas par transfection d'un plasmide contenant une version tronquée de TCF4 , dépourvue du site de liaison à la $\beta$ caténine, qui inhibe par compétition l'activité endogène des complexes TCF/ $\beta$-caténine, et dont l'expression est inductible par la doxycycline. Le choix de TCF-4 se justifie par son abondance dans les cellules coliques [4]. L'induction de dnTCF-4 par la doxycycline provoque un arrêt du cycle cellulaire en phase Gl. Une liste de 120 gènes ou EST (expressed sequence tag) dont l'expression est significativement diminuée après inhibition de la voie APC/ $\beta$-caténine/TCF peut être consultée sur le Tableau Sl de http://www.cell.com/cgi/content/full/ $111 / 2 / 241 / D C 1$. D'autre part 115 gènes ou EST sont surexprimés au moins 2,5 fois 
(Figure S2 et Tableau S2 du même site). L'analyse de l'expression spatiale de ces gènes dans la villosité intestinale est particulièrement intéressante: la muqueuse intestinale contient de nombreuses invaginations appelées cryptes. Au fond des cryptes nichent les cellules souches qui donnent naissance à une population de progéniteurs indifférenciés proliférant rapidement. Au cours de leur migration vers la lumière de l'intestin, ceux-ci arrêtent leur prolifération et se différencient en un des types cellulaires fonctionnels, puis sont éliminées à la surface de l'épithélium par un processus apoptotique. Ce cycle dure environ 3 à 5 jours, mais on sait peu de choses des mécanismes qui règlent cette migration et ordonnent la position relative des cellules dans l'épithélium intestinal. L'analyse en immunohistochimie des gènes différentiels identifiés par Van de Wetering et al. permet d'assigner quelques gènes dont l'expression est réduite après l'induction de dnTCF-4 (donc normalement activés par la voie wnt/wingless) à une localisation dans les cryptes coliques [6], et d'autres dont l'expression est activée par dnTCF-4 (donc normalement inactivés) à une localisation dans les cellules du sommet (normalement quiescentes) des cryptes intestinales. Ces observations indiquent que la voie wnt/wingless est active dans les cellules de la zone proliférative de l'épithélium colique normal, et inactive lorsque les cellules migrent vers le sommet de la crypte. De façon intéressante, cette distribution est également observée dans les lésions intestinales précoces.

Les auteurs se sont concentrés sur deux couples de gènes $[6,7], \mathrm{p} 21^{\mathrm{CIPl} / \mathrm{WAFl}}$ et le couple EphB/éphrine-B. P21 CIP1/WAFl, induite par l'activation de dnTCF-4, est un inhibiteur des couples cycline $\mathrm{A} / \mathrm{CDK} 2(\mathrm{Gl} \rightarrow \mathrm{S}$ ) et cycline $\varepsilon / C D K 4$, dont l'expression provoque l'arrêt du cycle cellulaire [8], et il est admis que ce phénomène est suffisant pour induire la différenciation cellulaire. Les auteurs ont montré que l'expression de $\mathrm{P} 21^{\mathrm{CIPl} / W A F l}$ était directement réprimée par C-MYC par fixation sur le promoteur de ce gène. Lorsque la voie de signalisation wnt/wingless est

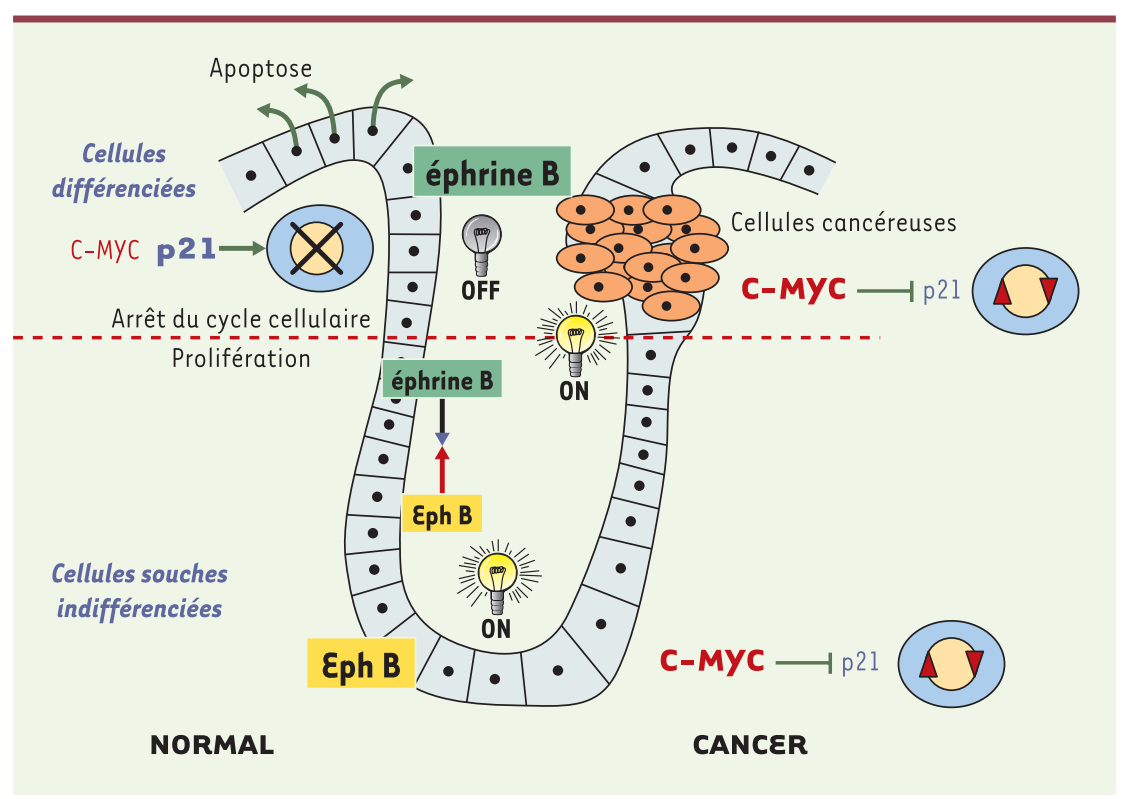

Figure 1. Voie Wnt/Wingless et prolifération cellulaire intestinale. Les positions « $O N$ » et «OFF» de la voie wnt/wingless sont représentées respectivement par une ampoule allumée ou éteinte. L'action de C-MYC et p2l sur le cycle cellulaire est également indiquée, de même que les quantités d'éphrine $B$ et de $\varepsilon$ ph $B$ produites, et leur action répulsive $(\rightarrow)$.

active, c-MYC est exprimée et inactive l'expression de P21 $1^{\text {CIP1/WAFl }}$ qui n'a alors aucun effet sur le cycle cellulaire. Lorsque la voie wnt/wingless est «OFF», la synthèse de P21 CIPl/WAFl n'est plus inhibée et peut arrêter le cycle cellulaire provoquant alors une différenciation (Figure 1). Dans les cancers, la voie wnt/wingless échappe au contrôle physiologique, si bien que les cellules continuent à se diviser et à se comporter comme des cellules progénitrices malgré leur localisation à la surface de l'épithélium. Il est à noter que la cycline $D l$, une des premières cibles décrites de la voie APC/ $\beta$-caténine/TCF [9] n'a pas été détectée dans le présent travail.

L'autre couple de gènes dont l'expression est modulée par l'induction de dnTCF-4 est le couple ephB/éphrine-B [7]. EphB est un récepteur à activité tyrosine kinase dont le ligand est l’éphrine-B. Les récepteurs Eph contrôlent la forme des cellules et leur migration, en remodelant le cytosquelette d'actine $(\rightarrow)$. Lorsque la voie wnt/wingless est active, la synthèse du récepteur

\section{$(\rightarrow) \mathrm{m} / \mathrm{s}$} $2000, n^{\circ} 2$ p. 241 EphB est induite, alors que c'est celle du ligand Ephrine-B qui est induite quand cette même voie est inactive. Les récepteurs EphB sont exprimés dans les cellules multipotentes progénitrices des cryptes intestinales, là où la voie wnt / wingless est active, et l'Éphrine-B est, quant à elle, exprimée là où la voie wnt/wingless est inactive, c'est-à-dire dans toutes les cellules à l'exception de celles du fond de la crypte. L'interaction entre les récepteurs $\varepsilon p h$ et leurs ligands Éphrines résulte le plus souvent en une répulsion des cellules. La voie wnt/wingless détermine donc, par son contrôle de l'expression des gènes ephB et ephrine-B le positionnement des cellules le long de l'axe des cryptes.

Pour résumer, il suffit de modifier légèrement les titres des deux articles commentés et de les mettre bout à bout: le 
complexe $\beta$-caténine/TCF-4 intervient dans le positionnement des cellules dans l'épithélium intestinal, et impose un phénotype de cellules souches aux cellules cancéreuses. $\diamond$

$\beta$-catenin and cell proliferation in normal and transformed intestinal cells

\section{RÉFÉRENCES}

1. Bellaïche $y$, Perrimon N. La voie de signalisation Wingless chez la drosophile. Med Sci 1997; 13: 166-74.

2. Romagnolo B. APC, $\beta$ caténine et cancer : les diaboliques. Med Sci 1997; 13: 872-3.

3. Behrens J, von Kries JP, Kuhl $M$, et al. Functional interaction of $\beta$-catenin with the transcription factor LEF-1. Nature 1996; 382: 638-42.
4. Korinek V, Barker N, Morin PJ, et al. Constitutive transcriptional activation by a $\beta$-catenin-Tcf complex in APC-/- colon carcinoma. Science 1997; 275: 1784-7.

5. He TC, Sparks AB, Rago C, et al. Identification of C-MYC as a target of the APC pathway. Science 1998; 281: 1509-12.

6. van de Wetering $M$, Sancho $\varepsilon$, Verweij $C$, et al. The $\beta$ catenin/TCF-4 complex imposes a crypt progenitor phenotype on colorectal cancer cells. Cell 2002; 111: 241-50.
7. Battle $\varepsilon$, Henderson JT, Beghtel $\mathrm{H}$, et al. $\beta$-catenin and TCF mediate cell positioning in the intestinal epithelium by controlling the expression of EphB/EphrinB. Cell 2002; 111: 251-63.

8. Tetsu 0, McCormick F. Betacatenin regulates expression of cyclin Dl in colon carcinoma cells. Nature 1999 ; $398:$ 422-6.

9. Pommier Y, Kohn KW. Cycle cellulaire et points de contrôle en oncologie : nouvelles cibles thérapeutiques. Med Sci $2003 ; 19$ : 173-86.

\section{NOUVELLE}

\section{Quand la diversité du répertoire des immunoglobulines augmente!}

Frédéric Ducancel

\author{
CEA, DIEP, UMR \\ CEA/bioMérieux, Bât. 152, CE \\ de Saclay, 91191 Gif-sur- \\ Yvette Cedex, France. \\ frederic.ducancel@cea.fr
}

> Depuis la mise en évidence du rôle des immunoglobulines (anticorps) dans la réponse immune, les scientifiques ont cherché à expliquer comment un répertoire limité d'anticorps peut interagir avec un nombre quasi infini d'antigènes. $\varepsilon n$ effet, les sites antigéniques reconnus par les anticorps peuvent être portés par toutes les molécules du monde vivant: sucres, lipides, acides nucléiques, petites molécules chimiques (haptènes), peptides, protéines; il peut aussi s'agir de structures moléculaires complexes telles que des protéines glycosylées. Cette diversité de reconnaissance apparaît plus grande encore par le fait qu'une même macromolécule peut exposer plusieurs sites antigéniques à sa surface, et que des anticorps sont également capables de reconnaître des composés organiques synthétiques, ainsi que des molécules du soi (dans les maladies auto-immunes). Les travaux de S.Tonegawa [1], qui lui valurent de recevoir le Prix Nobel de Médecine en 1987, constituèrent une avancée décisive dans la compréhension de l'origine de la diversité du répertoire des anticorps. Au cours du processus de sélection clonale, la combinaison d'un nombre fini de gènes, associée à l'imprécision de la jonction de ces différents gènes, à la combinatoire chaîne lourde-chaîne légère, et enfin aux mutations somatiques ponctuelles disséminées le long des régions variables des chaînes lourdes et légères, constituait jusqu'à maintenant l'ensemble des mécanismes à l'origine de la diversité du répertoire des immunoglobulines.

Toutefois, des travaux récents établissent qu'un niveau supplémentaire de variabilité du répertoire des anticorps existe ! $\varepsilon$ n effet, les résultats publiés dans la revue
Science [2, 3] par D. S. Tawfik et al. démontrent qu'une même molécule d'anticorps peut pré-exister sous plusieurs conformations structurales, chacune étant susceptible de reconnaître un antigène spécifique (Figure 1).

Un tel scénario avait été envisagé par Linus Pauling dès 1940 [4], et repris plus récemment sur la base de données expérimentales sur la cinétique de formation de complexes anticorps-antigène $[5,6]$. Ces derniers travaux suggéraient qu'un équilibre puisse exister entre plusieurs (au moins deux) conformations structurales (isomères) d'un anticorps unique, et que le ligand principal de cet anticorps reconnaissait préférentiellement un de ces isomères!

L'importance des travaux publiés par D.S. Tawfik et al. tient en ce qu'ils apportent des preuves expérimentales directes de l'existence d'un équilibre entre plu- 